\title{
INTERVALS OF CONTINUA WHICH ARE HILBERT CUBES
}

\author{
CARL EBERHART
}

\begin{abstract}
If $P$ is a subcontinuum of a metric continuum $X$, then by the interval of continua $\mathcal{C}(P, X)$ we mean the space of all subcontinua of $X$ which contain $P$ (with the Hausdorf metric). We show that $\mathcal{C}(P, X)$ is of ten homeomorphic with the Hilbert cube.
\end{abstract}

In what follows $X$ is a metric continuum and $\mathcal{C}(X)$ is the space of all subcontinua of $X$ with the Hausdorff metric. If $P \in \mathcal{C}(X)$, then by the interval of continua $\mathcal{C}(P, X)$ we mean the subspace of $\mathcal{C}(X)$ consisting of all $A \in \mathcal{C}(X)$ which contain $P$. In [3], Curtis and Schori have shown that if $X$ is locally connected and $X \backslash P$ is a nonvoid set containing no arc with interior, then the interval $\mathcal{C}(P, X)$ is homeomorphic with the Hilbert cube $Q$. In this note we will use a recent characterization of $Q$ due to Torunczyk [8] to show that $\mathcal{C}(P, X)$ is often homeomorphic with $Q$ without assuming $X$ is locally connected.

In order to state Torunczyk's theorem we need his definition of a $Z$-map. A closed subset $A$ of $X$ is called a $Z$-set in $X$ provided the identity map on $X$, $1_{X}$, can be approximated by maps $f: X \rightarrow X$ such that $f(X) \cap A=\varnothing$. A map $f: X \rightarrow X$ is a $Z$-map if $f(X)$ is a $Z$-set in $X$. The following remarkable result is a special case of Theorem 1 in Torunczyk's paper.

1. THEOREM [TORUNCZYK]. Suppose $X$ is an AR (absolute retract) such that $1_{X}$ can be approximated by $Z$-maps. Then $X \approx Q .(\approx$ means is homeomorphic with.)

This can be applied to intervals of continua because they are absolute retracts.

2. ThEOREM. $\mathcal{C}(P, X)$ is an $A R$.

Proof. It has been shown in [4] that $\mathcal{C}(P, X)$ is locally connected. Then using 4.3 in Kelley [6] and Lefschetz [7] it follows that $\mathcal{C}(P, X)$ is an ANR (absolute neighborhood retract). Now $\mathcal{C}(P, X)$ is contractible (to see this, let $f:[0,1] \rightarrow \mathcal{C}(P, X)$ be any map with $f(1)=P$ and $f(0)=X$ and note that the homotopy $H:[0,1] \times \mathcal{C}(P, X) \rightarrow \mathcal{C}(P, X)$ given by $H(t, A)=f(t) \cup A$ is a suitable contraction of $\mathcal{C}(P, X)$ to $\{X\})$, and so by Borsuk [1], $\mathcal{C}(P, X)$ is an AR.

Received by the editors August 1, 1977.

AMS (MOS) subject classifications (1970). Primary 57B20, 54F15.

Key words and phrases. Interval of continua, annular pair, cover.

○ American Mathematical Society 1978 
The following criterion provides a useful means of showing $\mathcal{C}(P, X) \approx Q$.

(*) Suppose for each $\varepsilon>0$ there is an $A \in \mathcal{C}(P, X)$ such that $A$ is within $\varepsilon$ of $P$ in the Hausdorff metric and $\mathcal{C}(A, X)$ is a Z-set in $\mathcal{C}(P, X)$. Then $\mathcal{C}(P, X)$ $\approx Q$.

(*) follows from (1) and (2) upon noting that the map $f: \mathcal{C}(P, X) \rightarrow$ $\mathcal{C}(A, X)$ given by $f(B)=A \cup B$ is a $Z$-map within $\varepsilon$ of $1_{\mathcal{C}(P, X)}$.

We use the device of "passing to the quotient space" to simplify our discussions. Let $X / P$ denote the quotient space obtained by identifying $P$ to a point. Then the interval $\mathcal{C}(P, X)$ is homeomorphic in a natural way with the interval $\mathcal{C}(\{P\}, X / P)$. So to show $\mathcal{C}(P, X) \approx Q$ it will suffice to assume $P$ is a singleton.

Call a pair $(C, B)$ of points in $\mathcal{C}(\{p\}, X)$ an annular pair provided $C \subseteq B$ and $B \backslash C$ is a nonvoid open set in $X$.

3. LEMMA. If $A \in \mathcal{C}(\{p\}, X)$ and for each $\delta>0$, there is an annular pair $(C, B)$ with $\operatorname{diam} B<\delta$ and $(B \backslash C) \cap A \neq \varnothing$, then $\mathcal{C}(A, X)$ is a Z-set in $\mathcal{C}(\{p\}, X)$.

Proof. Choose an annular pair $(C, B)$ with diam $B<\delta / 2$ and $(B \backslash C) \cap$ $A \neq \varnothing$, and consider the function $g$ defined on $\mathcal{C}(B, X)$ by $g(D)=D \backslash(B \backslash$ $C)$. Note that $g(D)$ is a closed subset of $X$ since $B \backslash C$ is open in $X$. In fact, $g(D) \in \mathcal{C}(\{p\}, X)$. To see this, let $x \in D \backslash B$ and let $K_{x}$ denote the closure of the component of $x$ in $D \backslash B$. Since $D$ is a continuum, we have that $K_{x} \cap B \neq \varnothing$. Also $K_{x} \subseteq g(D)$ since $g(D)$ is closed and contains $D \backslash B$. Hence $K_{x} \cap C \neq \varnothing$ and so $g(D)=C \cup\left(\cup_{x \in D \backslash B} K_{x}\right)$ is connected. Since $p \in D \cap C$ we have then that $g(D) \in \mathcal{C}(\{p\}, X)$ and since $(B \backslash C) \cap A \neq$ $\varnothing$ we have that $g(D) \notin \mathcal{C}(A, X)$. It follows quickly from the definition of the Hausdorff metric that $g$ is an isometry and that the Hausdorff distance from $D$ to $g(D)$ is less than $\delta / 2$.

To complete the argument let $f: \mathcal{C}(\{p\}, X) \rightarrow \mathcal{C}(B, X)$ be given by $f(D)=B \cup D$. Here again it follows from the definition of the Hausdorff metric that $d(D, f(D))<\delta / 2$ and $\dot{d}(D, E) \geqslant d(f(D), f(E))$. Thus $f$ is a continuous map within $\delta / 2$ of $1_{\mathcal{C}(\{p\}, X)}$. Now the composition $h: g \circ f$ is a map from $\mathcal{C}(\{p\}, X)$ to $\mathcal{C}(\{p\}, X)$ within $\delta$ of $1_{\mathcal{C}(\{p, X)}$ for which $h(\mathcal{C}(\{p\}, X)) \cap \mathcal{C}(A, X)=\varnothing$. So $\mathcal{C}(A, X)$ is a Z-set in $\mathcal{C}(\{p\}, X)$.

The following theorem shows that, in certain settings, whether or not $\mathcal{C}(\{p\}, X) \approx Q$ depends entirely on the nature of the space $X$ in a neighborhood of $p$.

4. Theorem. Suppose $X$ is locally connected at each point of an open set containing $p$. Then $\mathcal{C}(\{p\}, X) \approx Q$ if and only if $p$ is not in the interior (relative to $X$ ) of a finite graph in $X$.

Proof. If $p \in$ int $G$, where $G$ is a finite graph in $X$, then one can construct in a natural way an open $n$-cell in $C(\{p\}, X)$ about $\{p\}$, where $n$ is the order of $p$ in $G$. Conversely suppose $p$ does not lie in the interior of any finite graph in $X$. Let $\varepsilon>0$ be given. Choose a connected open set $U$ about $p$ whose 
diameter is less than $\varepsilon / 2$. We can assume that $X$ is locally connected at each point of the closure of $U$, which we will denote by $A$. To see that $\mathcal{C}(A, X)$ is a $Z$-set in $\mathcal{C}(\{p\}, X)$, let $\delta>0$ be given. Choose a connected open set $V$ about $p$ such that $\mathrm{cl} V \subseteq U$ and $\operatorname{diam} V<\delta / 2$. Using the local connectivity assumptions, cover the boundary of $V$ with a finite number of continua $C_{1}$, $C_{2}, \ldots, C_{n}$ so that $p \notin C_{i}$ for each $i$ and $\operatorname{diam}\left(\mathrm{cl} V \cup C_{1} \cup \cdots \cup C_{n}\right)<\delta$. Let $G$ be a finite graph in $V$ which contains $p$ and meets each $C_{i}$. $G$ exists because $V$ is arc-connected. Now let $B=\operatorname{cl} V \cup C_{1} \cup \cdots \cup C_{n}$ and let $C=G \cup C_{1} \cup C_{2} \cup \cdots \cup C_{n}$. Note that $B$ and $C$ are members of $\mathcal{C}(\{p\}$, $X)$ and $B \supseteq C$ with diam $B<\delta$. Also $B \backslash C$ is open in $X$ since $C$ contains the boundary of $V$ and $(B \backslash C) \cap A$ is nonvoid since $G$ does not contain $p$ in its interior in $X$. Thus $(C, B)$ is an annular pair which satisfies the hypothesis of (3). We conclude that $\mathcal{C}(A, X)$ is a $Z$-set in $\mathcal{C}(\{p\}, X)$.

We point out that the requirement that $X$ be locally connected at each point in a neighborhood of $p$ may be relaxed somewhat in (4). We illustrate with an example of a continuum $X$ in the plane which is not locally connected and yet $C(\{p\}, X) \approx Q$ for each $p \in X$.

Let $T_{0}$ be the convex hull of the points $(0,0),(0,1)$ and $(-1,1)$, and for each positive integer $n$, let $T_{n}$ be the convex hull of the points $(0,0),\left(1 / 2^{2 n}, 1\right)$ and $\left(1 / 2^{2 n+1}, 1\right)$. Let $X$ be the union of all these closed triangular regions. Then $X$ is a continuum which is locally connected at each point except along the edge $E$ of $T_{0}$ from $(0,0)$ to $(0,1)$. It is easy to see that (4) gives $\mathcal{C}(\{p\}, X) \approx Q$ for any point $p$ not in $E$. If $p \in E$ and $\varepsilon>0$, let $A_{\varepsilon}$ be the intersection of $T_{0}$ with the closed $\varepsilon$-disk about $p$ and let $B_{\varepsilon}$ be the boundary of $A_{\varepsilon}$. Then the pair $\left(B_{\varepsilon}, A_{\varepsilon}\right)$ is an annular pair as is easily verified. From (3) we conclude that $\mathcal{C}\left(A_{\delta}, X\right)$ is a $Z$-set in $\mathcal{C}(\{p\}, X)$. Then by $(*), \mathcal{C}(\{p\}, X) \approx$ $Q$.

The result in (4) can be restated: Suppose $X$ is locally connected in a neighborhood of $p$. Then $\mathcal{C}(\{p\}, X) \approx Q$ if and only if $\mathcal{C}(\{p\}, X)$ is infinite dimensional at $\{p\}$.

In the remainder we give another method for showing that $\mathcal{C}(\{p\}, X) \approx Q$ which will, for example, show that if $X$ is the Cantor fan (cone over the Cantor set), then $\mathcal{C}(\{p\}, X) \approx Q$ where $p$ is the vertex of the cone. The criterion which applies here is an immediate consequence of (1) and (2).

(**) Suppose for each $\varepsilon>0$, there is an $A \in \mathcal{C}(\{p\}, X)$ and a map $f$ : $\mathcal{C}(\{p\}, X) \rightarrow \mathcal{C}(\{p\}, A)$ such that $\mathcal{C}(\{p\}, A)$ is a $Z$-set in $\mathcal{C}(\{p\}, X)$ and $f$ is within $\varepsilon$ of the identity on $\mathcal{C}(\{p\}, X)$. Then $\mathcal{C}(\{p\}, X) \approx Q$.

If $A \in \mathcal{C}(\{p\}, X)$, then $A$ is said to cover $p$ provided the interior of $\mathcal{C}(\{p\}, A)$ in $\mathcal{C}(\{p\}, X)$ contains $\{p\}$.

5. Lemma. Suppose $A \in \mathcal{C}(\{p\}, X)$ does not cover $p$. Then $\mathcal{C}(\{p\}, A)$ is a $Z$-set in $\mathrm{C}(\{p\}, X)$.

Proof. Let $\varepsilon>0$. Since $A$ does not cover $p$ there is a $B \in \mathcal{C}(\{p\}, X)$ such that $\operatorname{diam} B<\varepsilon$ and $B \cap(X \backslash A) \neq \varnothing$. Then the map $f: \mathcal{C}(\{p\}, X) \rightarrow$ $\mathcal{C}(B, X)$ given by $f(D)=B \cup D$ is $\varepsilon$-close to the identity on $\mathcal{C}(\{p\}, X)$ since 
diam $B<\varepsilon$. The condition $B \cap X \backslash A \neq \varnothing$ implies the image of $f$ misses $\mathcal{C}(\{p\}, A)$. So $\mathcal{C}(\{p\}, A)$ is a $Z$-set in $\mathcal{C}(\{p\}, X)$.

6. THEOREM. Suppose the identity on $X$ can be approximated by maps $f$ such that $f(p)=p$ and $f(X)$ does not cover $p$. Then $C(\{p\}, X) \approx Q$.

Proof. Since $f(p)=p$, the induced map $\bar{f}$ on $\mathcal{C}(X)$ given by $\bar{f}(A)=$ $\{f(x) \mid x \in A\}$ takes $\mathcal{C}(\{p\}, X)$ into $\mathcal{C}(\{p\}, f(X))$. Clearly if $d\left(f, 1_{X}\right)<\varepsilon$, then $d\left(\bar{f}, 1_{\mathcal{C}(\{p\}, X)}\right)<\varepsilon$. Further if $f(X)$ does not cover $p$, then the image of $\bar{f}$, being a closed subset of the $Z$-set $\mathcal{C}(\{p\}, f(X))$, is a $Z$-set in $\mathcal{C}(\{p\}, X)$. Now apply (**).

This theorem has numerous applications.

7. Corollary. Suppose $X$ is a compact metric space ( $X$ not assumed connected here) such that the identity map on $X$ can be approximated by maps $f$ : $X \rightarrow X$ such that $f(X) \neq X$. Then $\mathcal{C}(\{p\}$, Cone $X) \approx Q$, where $p$ is the vertex of Cone $X$, the cone over $X$.

Proof. Embed $X$ in $Q$ and realize Cone $X$ as the subspace $\{(t, t x) \mid t \in$ $[0,1], x \in X\}$ of $[0,1] \times Q$. Now any map $f: X \rightarrow X$ induces a map $f^{\prime}:$ Cone $X \rightarrow$ Cone $X$ given by $f^{\prime}(t, t x)=(t, t f(x))$. Note that $f^{\prime}(p)=p$ where $p=(0,0, \ldots)$ is the vertex of Cone $X$ and that $d\left(f^{\prime}, 1_{\text {Cone } X}\right)=d\left(f, 1_{X}\right)$. Also if $f(X) \neq X$, then $f^{\prime}($ Cone $X)$ does not cover $p$. Hence (6) applies.

Thus as mentioned above, (7) can be used to show that $C(\{p\}, X) \approx Q$ when $X$ is the Cantor fan and $p$ is the vertex. In this case we can also use (8) below.

We can of ten use (6) to show that $C(p, X) \approx Q$ when $X$ is a dendroid; that is, a hereditarily unicoherent arc-connected continuum. For example, let $X$ be the continuum in the plane consisting of the union of all segments from $(0,0)$ to $(1, t)$ or from $(0,1)$ to $(-t, 0)$ where $t$ runs over the Cantor ternary set $C$. To see that $\mathcal{C}(\{(0,0)\}, X) \approx Q$, let $f_{n}: X \rightarrow X$ be defined by $f_{n}((r, r \cdot t))=$ $(r, 0)$ if $r \in(0,1]$ and $t \in C \cap\left[0,1 / 3^{n}\right]$ and let $f_{n}(x)=x$ otherwise. Then $f_{n}$ is a retraction which moves no point more than $1 / 3^{n}$. Further $f_{n}(X)$ does not cover $(0,0)$, since the segment $A_{\varepsilon}$ from $\left(\varepsilon, \varepsilon / 3^{n}\right)$ to $(0,0)$ meets $X \backslash f_{n}(X)$ for all $\varepsilon>0$. Since the maps $f_{n}$ approximate $1_{X},(6)$ applies.

If $X$ is a dendroid smooth at $p$ (see [2] for the definition of smooth dendroid) we have an analogue of (4).

8. TheOrem. Suppose $X$ is a dendroid smooth at $p$. Then $\mathcal{C}(\{p\}, X) \approx Q$ if and only if $p$ is not in the interior of a finite tree in $X$.

Proof. J. B. Fugate has shown that for each $\varepsilon>0$, there is an $\varepsilon$-retraction from $X$ onto a finite tree $T$ in $X$ [5]. In his construction, $p \in T$. The image of such an $\varepsilon$-retraction cannot cover $p$. This is because $X$ is locally connected at $p$ and, by assumption, $p \notin$ int $T$. Hence $\mathcal{C}(\{p\}, X) \approx Q$. 


\section{REFERENCES}

1. K. Borsuk, Theory of retracts, Monografie Mat., vol. 44, Polish Scientific Publishers, Warszawa, Poland, 1967.

2. J. J. Charatonik and C. A. Eberhart, On smooth dendroids, Fund. Math. 67 (1970), 297-322.

3. D. W. Curtis and R. M. Schori, $2^{X}$ and $C(X)$ are homeomorphic to the Hilbert cube, Bull. Amer. Math. Soc. 80 (1974), 927-931.

4. Carl Eberhart, Contimua with locally connected Whitney continua, Houston J. Math. (to appear).

5. J. B. Fugate, Small retractions of smooth dendroids onto trees, Fund. Math. 71 (1971), 256-262.

6. J. L. Kelley, Hyperspaces of a continuum, Trans. Amer. Math. Soc. 52 (1942), 22-36.

7. S. Lefschetz, Topology, Amer. Math. Soc. Colloq. Publ., vol. 12, Amer. Math. Soc., Providence, R.I., 1930 (revised as Algebraic topology, vol. 27, 1942; reprinted 1974).

8. H. Torunczyk, On CE-images of the Hilbert cube and characterization of Q-manifolds (preprint).

Department of Mathematics, University of Kentucky, Lexington, Kentucky 40506 